\title{
Interpretation and value of MR CSF flow studies for paediatric neurosurgery
}

\author{
S Mbonane, S Andronikou \\ Department of Diagnostic Radiology, Faculty of Health Sciences, University of the Witwatersrand, Johannesburg \\ S Mbonane, MB ChB, Dip HIV Man (SA) \\ $\mathrm{S}$ Andronikou, MB BCh, FCRad, FRCR (Lond), PhD
}

Corresponding author: S Mbonane (Nkalankala@yahoo.com)

\begin{abstract}
Imaging techniques may be underutilised when clinicians are unaware of the technique or do not recognise its potential. Phase-contrast MR imaging (PC-MRI) is a rapid, simple and non-invasive technique that is sensitive to CSF flow. It demonstrates a mechanical coupling between cerebral blood and CSF flow throughout the cardiac cycle. Neurosurgeons should be able to request this procedure routinely as part of an MRI request. This paper gives an overview of the indications, technical requirements, technique and interpretation, using image examples. Indications for CSF flow studies in children include assessment and functionality of shunt treatment in patients with hydrocephalus; hydrocephalus associated with achondroplasia; Chiari I malformation; confirmation of aqueductal stenosis; and determining patency of a third ventriculostomy.
\end{abstract}

S Afr J Rad 2013;17(1):26-29. DOI:10.7196/SAJR.747

New techniques in one discipline are often slow to filter to another. In particular, imaging techniques may be underutilised because the end user is unaware of the technique, does not recognise the potential, or is led to believe that it is an unavailable or unreasonable service.

Phase-contrast MR imaging (PC-MRI) is a rapid, simple and noninvasive technique, and is sensitive to CSF flow. ${ }^{[1,2]}$ It has been available for some time, and been used in the past decade in the evaluation of cranial and spinal CSF flow, demonstrating a mechanical 'coupling between cerebral blood and CSF flows throughout the cardiac cycle and the temporal coordinated succession of these flows' in normal people. ${ }^{[1]}$ The technique may lead to a better understanding of the pathophysiological basis of diseases with dysfunction of CSF flow. ${ }^{[2]}$

Neurosurgeons, neurologists and in particular those working with children should be able to request this procedure from any MR imaging department, and should also see this sequence routinely as part of the battery of sequences provided for certain indications. This paper aims to familiarise clinicians and radiologists working with children with the indications and technique, and also discusses interpretation of the information provided.

\section{Indications for CSF flow studies in paediatrics}

There is good reason to demonstrate CSF flow dynamics routinely in children undergoing MRI because their evolving and maturing brain creates a changing volume of CSF in relation to the brain parenchyma. The absence of flow-void signal intensity on sagittal T2 MR images has been proposed as a direct sign of CSF pathway obstruction, but the flow-void signal intensity depends on numerous parameters that make it unreliable (Fig. 1a). ${ }^{[1]}$

There are also some clear indications for CSF flow studies without which routine MRI offers only limited and mainly structural information. It may be useful for better assessment and functionality of shunt treatment in patients with hydrocephalus. ${ }^{[2]}$ One of the
Figs 1(a - e) (structural sagittal MRI imaging of CSF flow) follow below.

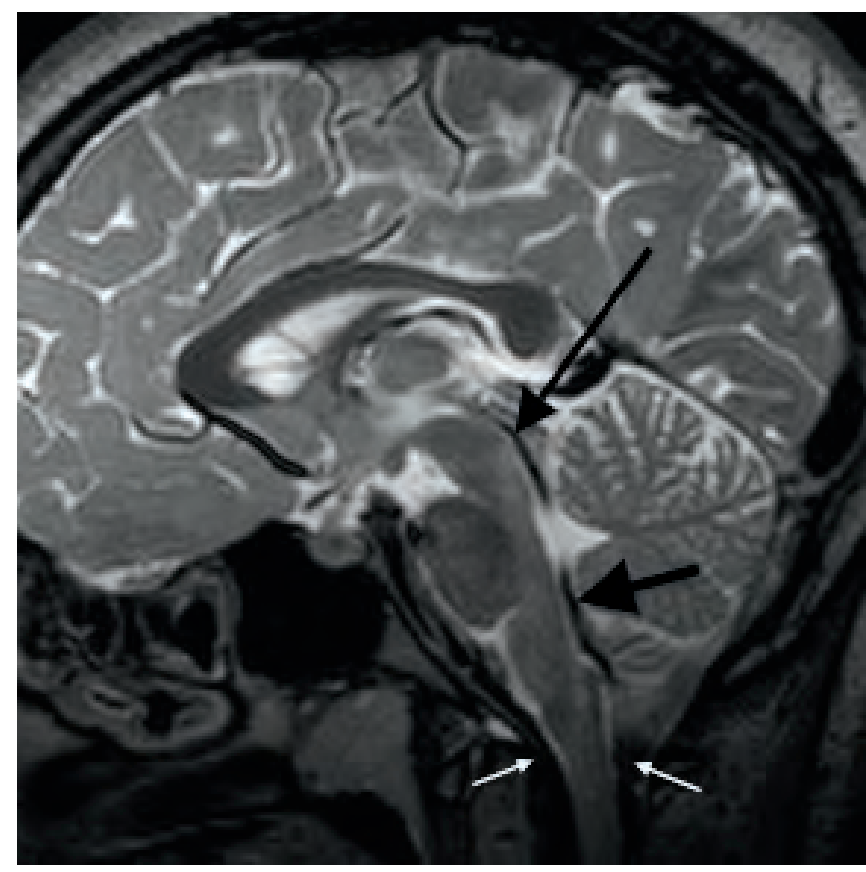

Fig. 1(a). Normal sagittal T2-weighted MRI demonstrates CSF flow-related signal void at the aqueduct of Sylvius (long black arrow), foramen of Magendie (thick black arrow), and foramen magnum (white arrows).

more specific uses of CSF flow studies is to gain information relating to hydrocephalus associated with achondroplasia (Fig. 1b). ${ }^{[3]}$ CSF studies also provide a better understanding of the pathophysiology of Chiari I malformation regarding the spatial and temporal foramen magnum CSF flow patterns (Figs 1c - e). Comparison of CSF flow patterns between symptomatic and asymptomatic patients, those with or without syringomyelia, and those with syringomyelia but 


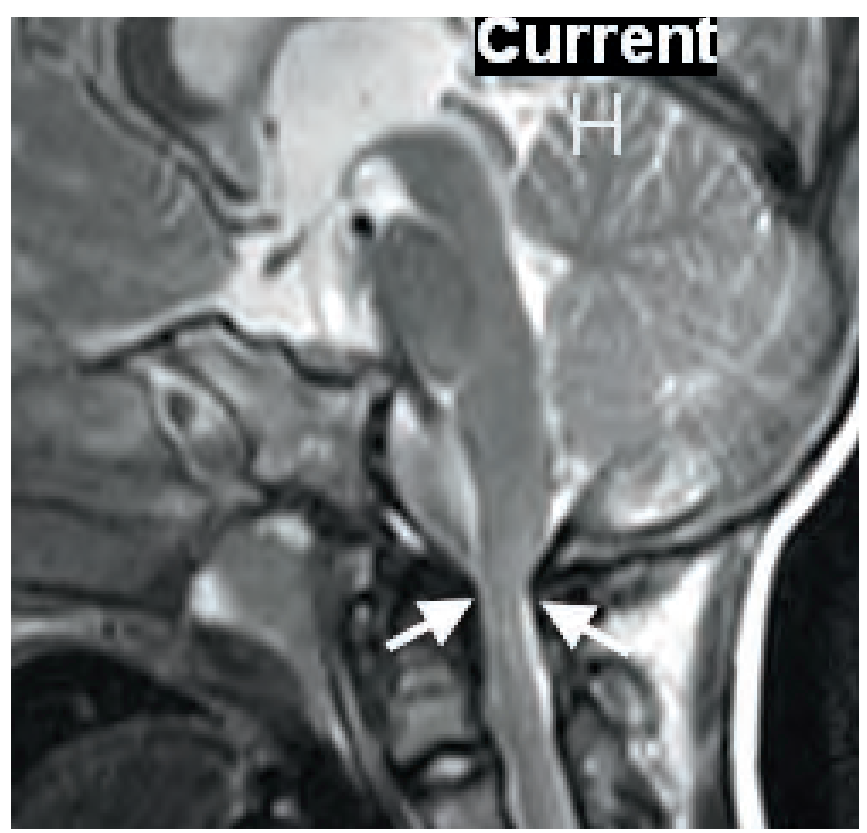

Fig. 1(b). Sagittal T2 view in a child with achondroplasia demonstrates a funnel-shaped posterior fossa with lack of CSF signal and flow void at the foramen magnum (arrows).

with or without tonsillar herniation, is possible. ${ }^{[4]}$ PC-MRI is able to confirm aqueductal stenosis suspected on morphological sequences and is sensitive even to slow CSF flows at the aqueductal level. ${ }^{[1]}$ Efforts to visualise the patency of a third ventriculostomy begin with the detection of a flow void in the floor of the third ventricle on T2-weighted MR images and later confirmation with the use of cine 2-dimensional (2D) phase-contrast (PC) MR images. ${ }^{[5]}$ Patency on cine PC MR images correlates well with clinical prognosis, but whether

\section{Table 1. Indications for performing CSF flow studies in children}

Hydrocephalus $^{[2]}$

- aqueductal stenosis

- specific causes of flow obstruction at the craniocervical junction:

- achondroplasia $^{[3]}$

- Chiari I malformation ${ }^{[6]}$

- patency of third ventriculostomy. ${ }^{[5]}$ patency as perceived on MR images represents actual anatomic patency has never been proved. ${ }^{[5]}$

\section{Technical requirements}

No special hardware is required, and imaging can be performed on all modern $1.5 \mathrm{~T}$ scanners equipped with standard software and phase contrast magnetic resonance (PCMR) capabilities and analysis packages ${ }^{[6]}$ Imaging requires routine localiser images, sagittal T1- and T2-weighted images of the cervical spine, and a series of head and axial spine images as performed for clinical indications. ${ }^{[6]}$ Axial and sagittal PCMR images are acquired additionally. ${ }^{[6]}$ The images are gated to the cardiac cycle by electrocardiography, which is available routinely on modern scanners. ${ }^{[6]}$

\section{PCMR technique and acquisitions}

Time-resolved 2D PC-MRI with velocity encoding in one spatial direction is the method of choice for investigation of CSF flow. ${ }^{[2]}$ For sagittal PCMR, the midline sagittal plane is chosen from the regular imaging. ${ }^{[6]}$ For the axial images, a plane transverse to the axis of the spinal canal immediately below the tonsillar tips is selected most commonly, but this can be performed in any area of interest e.g. the aqueductal level or the level of the foramen magnum. ${ }^{[6]}$ (Recommended acquisition parameters are flip angle $20^{\circ}$; TR/TE 20/5 ms; section thickness $5 \mathrm{~mm}$; FOV $180 \mathrm{~mm}$; matrix $256 \times 256$; and encoding velocity $10 \mathrm{~cm} / \mathrm{s}$. $)^{[6]}$ In each subject, the CSF flow images can first be acquired with the patient's neck in a neutral position, then in a flexed, and finally in an extended position. ${ }^{[6]}$

\section{Interpretation of the information provided}

Intracranial blood and CSF 'flush' and 'fill' flows through the cardiac cycle are initiated by the systolic intracerebral arterial inflow. Studies, using PC-MRI, proposed a dynamic model for a mechanical coupling between blood and CSF intracranial flows. The systolic arterial fill flow peak in the carotid arteries results in an instantaneous increase in intracranial pressure. The first and fastest way to decrease intracranial pressure is a large CSF venting from the subarachnoid spaces, which drops the cerebral subarachnoid space pressure. Then flush flows occur in the cerebral venous and aqueductal CSF compartments. An imbalance in this mechanical coupling is presumed to be responsible for pathological cerebral states, such as normal pressure hydrocephalus (NPH) or Alzheimer disease. ${ }^{[1]}$

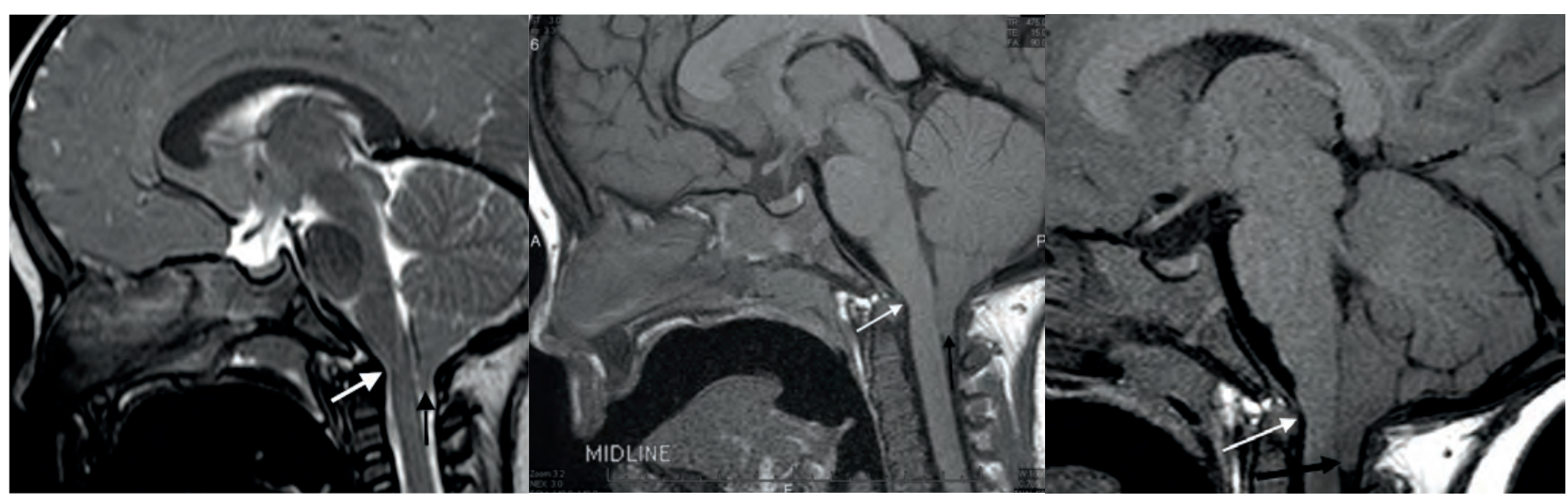

Fig. 1(c-e). Sagittal (c) T2- and (d and e) T1-weighted MRI demonstrates tonsillar herniation in keeping with a Chiari I malformation (black arrow). There is resultant crowding of the foramen magnum with compression of the anterior CSF space (white arrow). 
Line graphs demonstrating different time courses for CSF flow use the Y-axis to show the magnitude of flow in positive and negative directions in $\mathrm{ml} / \mathrm{s}$; the X-axis shows time as a decimal fraction of the cardiac cycle. Time courses may illustrate CSF flowing in a sinusoidal manner, or in a less symmetric manner. The maximal velocity may be in a positive or a negative direction (systolic flow). A change in direction (e.g. from a positive to a negative flow) then demonstrates the diastolic flow. Systolic flow lasts a shorter time and has a greater magnitude than diastolic flow. Net flow during the cardiac cycle is zero (Figs $2 \mathrm{a}-\mathrm{d}$ and $3 \mathrm{a}-\mathrm{d}$ ). ${ }^{[7]}$

CSF flow is classified as hypermotile if it shows increased dynamics, and as hypomotile if it shows attenuated dynamics. ${ }^{[2]}$ The maximum

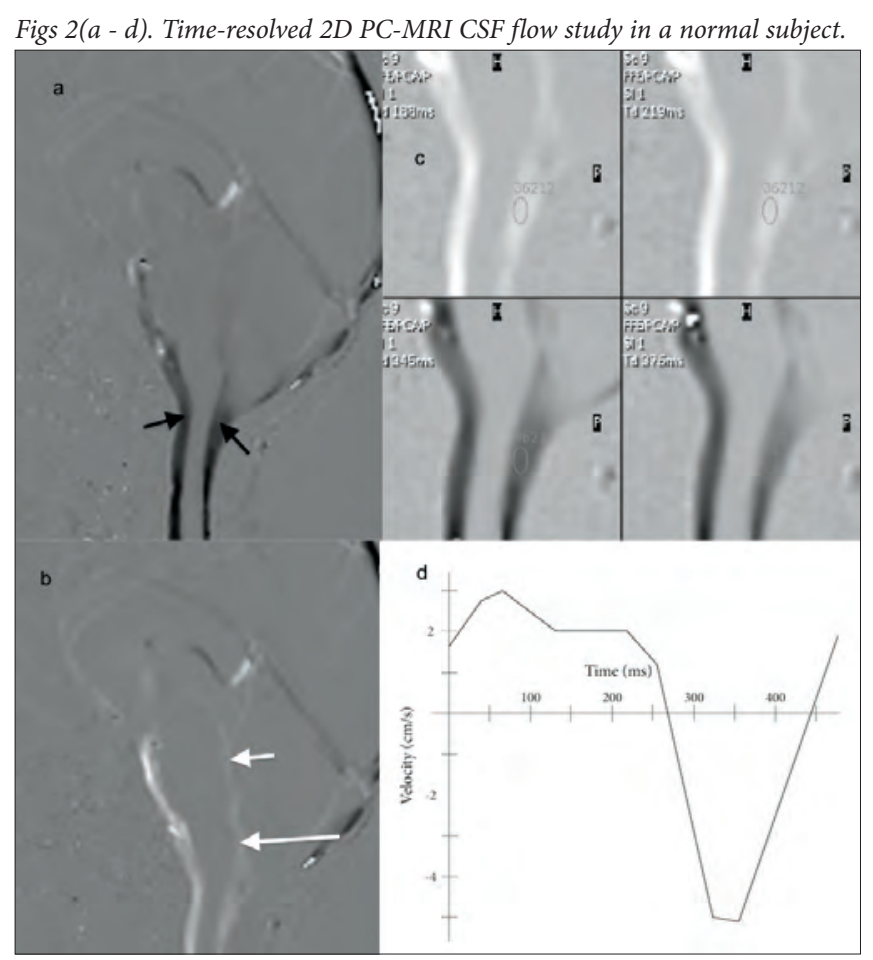

Fig. 2 (a). Single sagittal midline slice of CSF flow during systole is demonstrated as low signal, anterior and posterior at the foramen magnum (black arrows). There is also a subtle CSF flow at the foramen of Magendie and aqueduct of Sylvius. (b) Single sagittal midline slice during diastole demonstrates CSF flow as a high signal at the foramen magnum and more obvious flow at the aqueduct of Sylvius (short white arrow) and foramen of Magendie (long white arrow). (c) CSF flow sequence through the cardiac cycle represented in the midline sagittal view at the foramen magnum that demonstrates the bidirectional flow as high and low signal in turn. The oval region of interest (ROI) has been placed in the normal posterior CSF space on all images throughout the cardiac cycle to generate the graphic representation seen in $2 d$. (d) The graphic representation of CSF flow through the cardiac cycle is generated using a ROI placed on all slices by the investigator in any part where CSF is identified (on the sagittal or axial images). The graph generated reflects velocity in $\mathrm{cm} / \mathrm{s}$ on the Y-axis and time in $\mathrm{ms}$ on the $X$-axis. In this normal study, the diastolic flow is represented above the $X$-axis and has a maximum velocity of $3 \mathrm{~cm} / \mathrm{s}$ and lasts for $260 \mathrm{~ms}$. Systole in this instance is represented below the baseline and shows a maximum velocity of $5 \mathrm{~cm} / \mathrm{s}$; it lasts for a shorter period than diastole - in this instance $190 \mathrm{~ms}$. Note that the black or white signal for systole and diastole or representation above or below the baseline on the graph is only relevant for demonstrating the direction of flow, and not the magnitude.

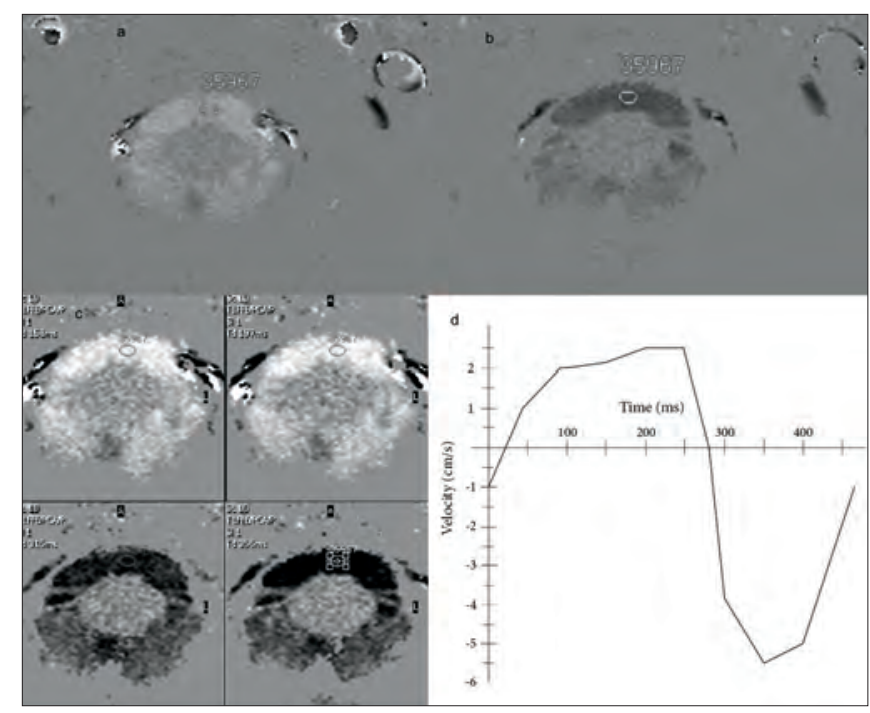

Fig. 3. Axial CSF flow study at the foramen magnum demonstrating normal bidirectional flow with graphic representation of a ROI in the anterior thecal sac.

peak value (v peak) and the mean value ( $\mathrm{v}$ mean) of the magnitude of the velocity vector can be determined directly from the line graphs produced. $^{[2]}$

\section{MR data analysis in achondroplasia}

In achondroplasia, the cine mode MR imaging demonstrates CSF flow disturbance at the cervicospinal junction resulting from foramen magnum stenosis and medullary compression. ${ }^{[3]}$ Attenuated flow is often noted between the suboccipital subarachnoid space and the craniocervical junction, which improves after craniotomy.

\section{MR data analysis in Chiari I malformation}

In children with symptomatic Chiari I malformation, even though bulk flow or velocity is often normal, there is marked heterogeneity of flow at the foramen magnum. ${ }^{[4]}$ This manifests in several ways: $(i)$ an increase in cephalad and caudad peak velocities; (ii) spatial inhomogeneity in velocities; (iii) simultaneous bidirectional flow; and (iv) substantial net craniad or caudad flows within particular voxels and sub regions during the cardiac cycle (Figs $4 a-d$ and $5 a-f) \cdot{ }^{[4]}$

\section{Aqueductal stenosis}

Diagnosis of aqueductal stenosis may be difficult because of the various clinical, aetiological and radiological features. It is also difficult to determine complete obstruction of the aqueduct, which is relevant because third ventriculostomy is most successful in true obstructive hydrocephalus. The absence of flow void signal intensity on standard sagittal T2 MR images is unreliable as it depends on several parameters (aqueductal diameter, CSF velocity, section thickness) and may be weak or absent if the aqueduct is physiologically narrow but not obstructed. ${ }^{[1]}$ PC-MRI can confirm the diagnosis of CSF flow blockage at the aqueductal level in a reliable, reproducible and rapid manner. ${ }^{[1]}$ In addition, even though fourth ventricular stroke volumes are similar to normal patients, the CSF flush peak occurs significantly earlier in patients with aqueductal stenosis. ${ }^{[1]}$ There is also a $50 \%$ reduction of atrioventricular delay (representing the latency between the 


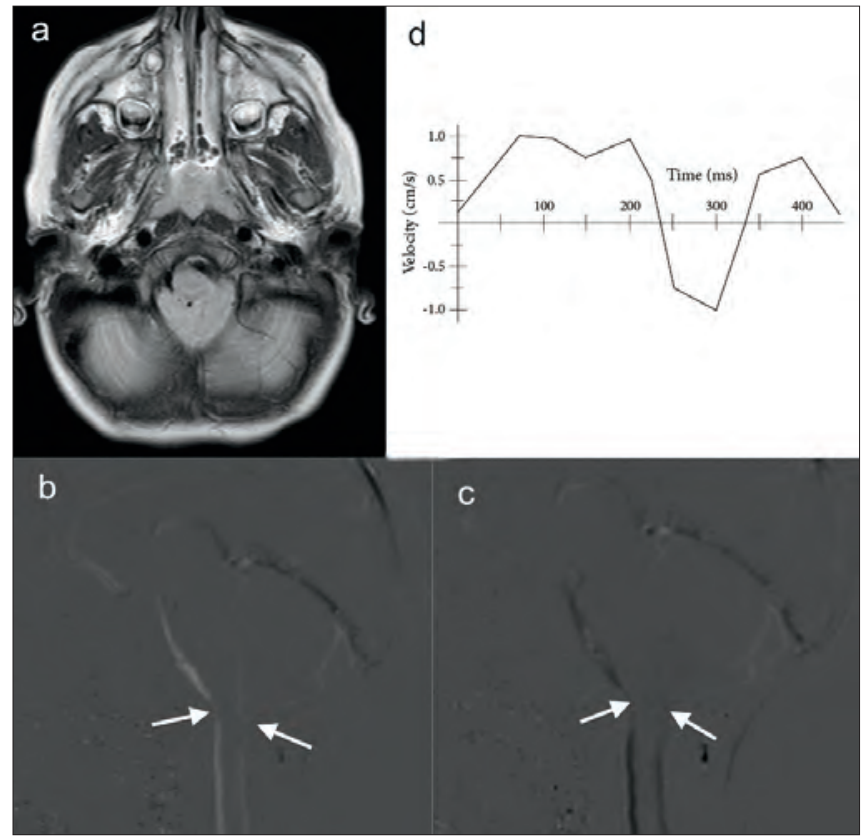

Fig. 4. A patient with Chiari I demonstrates (a) crowding of the foramen magnum by cerebellar tonsillar descent with (b) bi-directional flow that is severely restricted at the foramen magnum (white arrows). Graphic representation (c) demonstrates low peak velocities of equal volume during systole and diastole as well as short systolic duration.

arterial systolic inflow peak and the outflow venous peak) in patients with aqueductal stenosis. ${ }^{[1]}$

\section{MR interpretation post ventriculostomy}

Cine PC-MRI for third ventriculostomy should show patency by demonstrating continuous flow from the third ventricle to the pre-pontine cistern through a small discontinuation in the floor of the third ventricle..$^{[5]}$

\section{Postoperative follow-up}

After posterior fossa decompression in Chiari I, the severity of flow abnormalities decreases. ${ }^{[4]}$

\section{Recommendations}

PC-MRI is an available and relatively simple method of determining true CSF flow, and that also provides directional and additional physiological information. In paediatric practice, it is an extremely useful tool for evaluating causes of hydrocephalus and CSF obstruction at the level of the aqueduct, the fourth ventricle, the outlet foramina of the fourth ventricle and the foramen magnum. It should form part of the routine arsenal of sequences performed in children with clinical or radiological suspicion of CSF flow obstruction, and radiologists must be familiar with determining the level at which imaging should be performed and interpreting both the images and graphic physiological representation for directing management.

1. Stoquart-El S, Sankari P, Lehmann C, et al. Phase-contrast MR imaging support for the diagnosis of aqueductal stenosis. J Neuroradiol 2009;30:209-214.

2. Stadlbauer A, Salomonowitz E, Brenneis C, et al. Magnetic resonance velocity mapping of 3D cerebrospinal fluid_flow dynamics in hydrocephalus: preliminary results. Eur Radiol 2012;22(1):232-242. DOI10.1007/00330-011-2247-7.

3. Miyamoto J, Tatsuzawa K, Sasamija H, Mineuria K. Usefulness of phase contrast cine mode magnetic resonance imaging for surgical decision making in patients with hydrocephalus combined with achondroplasia. Neurol Med Chir (Tokyo) 2010;50:1116-1118.

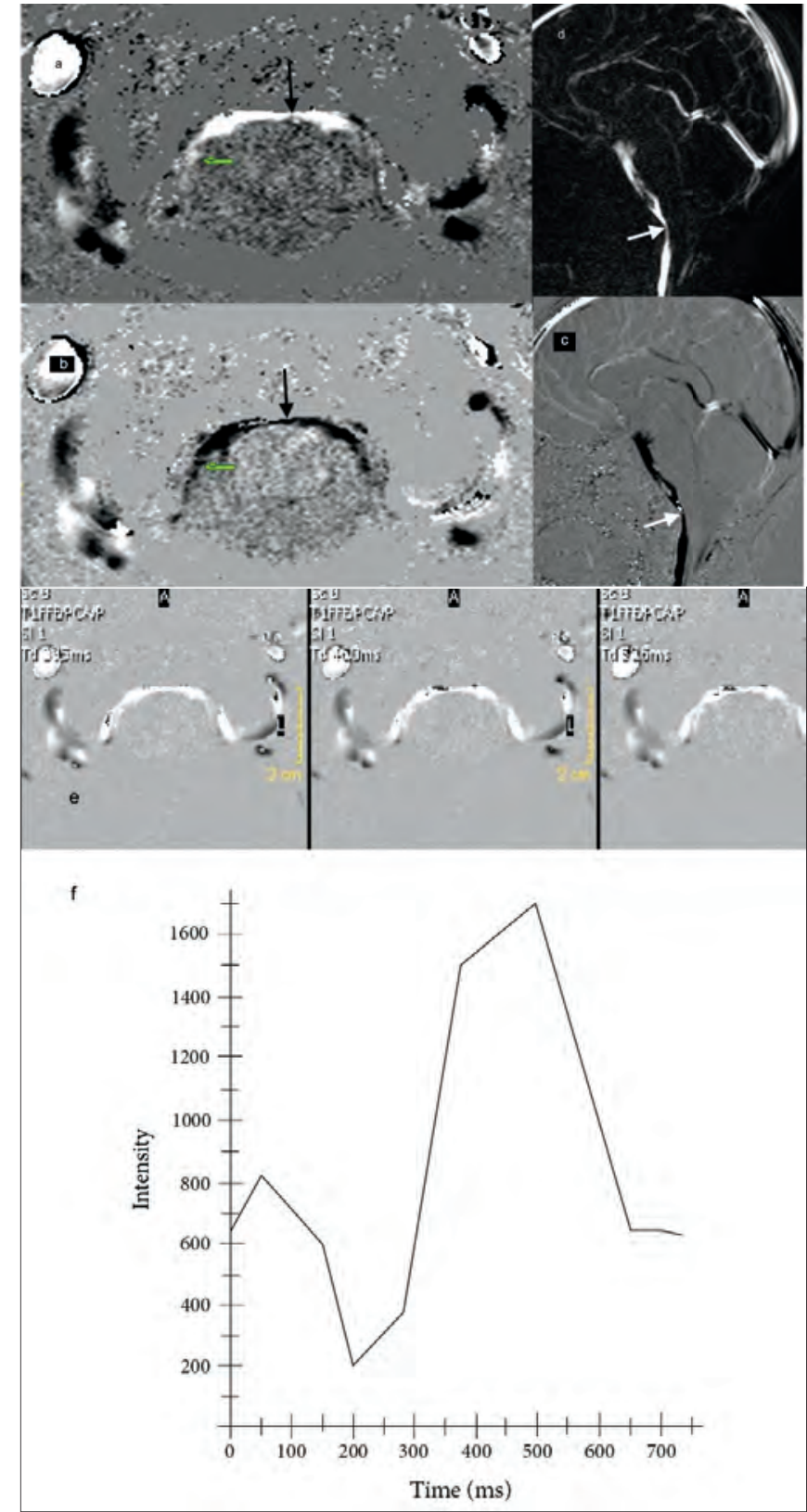

Fig. 5. (a - d) Axial phase contrast CSF flow study demonstrating baseline and follow-up imaging in a patient with Chiari I malformation. (e - f) Baseline axial and sagittal PC-MRI demonstrates absence of flow posteriorly at the foramen magnum, and limited flow at the midline anteriorly (black arrow). Follow-up imaging demonstrates change in CSF flow to a unidirectional pattern represented as only high signal on the sagittal PC-MRI and with high flow velocity above the baseline on the graphic representation.

4. Iskandar J, Quigley M, Haughton M. Foramen magnum cerebrospinal fluid flow characteristics in children with Chiari I malformation before and after craniocervical decompression. J Neurosurg Pediatr 2004; 01:169-178.

5. Fukuhara T, Vorster S, Ruggieri P, Luciano G. Third ventriculostomy patency: Comparison of findings at cine phase-contrast MR imaging and at direct exploration. Am J Neuroradiol 2009;20:1560-1566

6. Bhadelia R, Frederick E, Patz S, Dubey P. Cough-associated headache in patients with Chiari I malformation: CSF flow analysis by means of cine phase-contrast MR imaging. J Neuroradiol 2011;32:739-742.

7. Hentschel S, Mardal K, Løvgren A, Linge S, Haughton V. Characterization of cyclic CSF flow in the foramen magnum and upper cervical spinal canal with MR flow imaging and computational fluid dynamics. J Neuroradiol 2010;31:997-1002. 